\title{
An easy access to supramolecular gels of tolfenamic acid
}

\author{
$\underline{\text { Rumana Parveen }}^{1}$, Parthasarathi Dastidar $^{1}$ \\ ${ }^{1}$ Organic Chemistry, IACS, Kolkata, India \\ E-mail: ocrp@iacs.res.in
}

An Easy Access to Supramolecular Gels of Tolfenamic Acid

Rumana Parveen and Parthasarathi Dastidar

Department of Organic Chemistry, Indian Association for the Cultivation of Science, 2A \& 2B Raja S. C. Mullick Road, Kolkata-700032, India, E-mail: ocrp@iacs.res.in

Supramolecular gels are common place in Nature and our everyday life. Various studies (microscopy, X-ray diffraction etc.) indicate that the gelator molecules form a 3D network structure (self-assembled fibrillar networks or SAFiNs) from 1D fibers (resulting from anisotropic self-assembly of the gelator molecules sustained by various supramolecular or non-covalent interactions) via entanglement within which the solvent molecules are immobilized resulting in gel. Research activities pertaining to converting drug molecules into supramolecular gels has been intensified recently because of the various impediments such as synthetic limitation of carrier molecule, loading drug molecule and its sustain release, cytoxicity of the carrier molecule etc. associated with the conventional drug delivery system. Self- delivery1 is an alternative option where modification of the drug molecule in such a way that the drug may be effectively delivered to the affected site without the help of a carrier molecule. Topical gel formation is one of the several possible routes for self-delivery. Time-consuming nontrivial covalent modifications of the drug molecules are the main drawbacks associated with topical gel formulation. The present work aims at generating a new series of supramolecular gelators derived from a non-steroidal-anti-inflammatory drug (NSAID) - tolfenamic acid and its $\beta$-alanine derivative for developing drug delivery system that works in self-delivery fashion. A new series of primary ammonium monocarboxylate (PAM) salts of a non-steroidal-anti-Inflammatory-drug (NSAID) namely Tolfenamic acid (TA) and its $\beta$-alanine derivative was generated; nearly $67 \%$ of the salts in the series showed gelling ability with various solvents including water (biogenic solvent) and methyl salicylate (typically used for topical gel formulations). Gels were characterized by rheology, electron microscopy etc. Structure-property correlation based on single crystal and powder X-ray diffraction data of several gelator and non-gelator salts revealed intriguing insights. Studies (in vitro) with an aggressive human breast cancer cell line (MDA-MB-231) with L-tyrosine methyl ester salt of TA (S7) revealed that the hydrogelator salt was effective in killing the cancer cells more efficiently than the mother drug TA (MTT assay), displayed better anti-inflammatory activity as compared to that TA (PGE2 assay), could be internalized within the cancer cells as revealed by fluorescence microscopy and inhibited effectively migration of the cancer cells. Thus the easily accessible ambidextrous gelator salt S7 can be used for dual purpose- anti-inflammatory topical gel as well as anti-cancer agent.

1. Xu, B. et al. (2013) J. Am. Chem. Soc. 135, 542.

2. Dastidar, P. et al. (2017) Chem. Asian J. DOI: 10.1002/asia.201700049

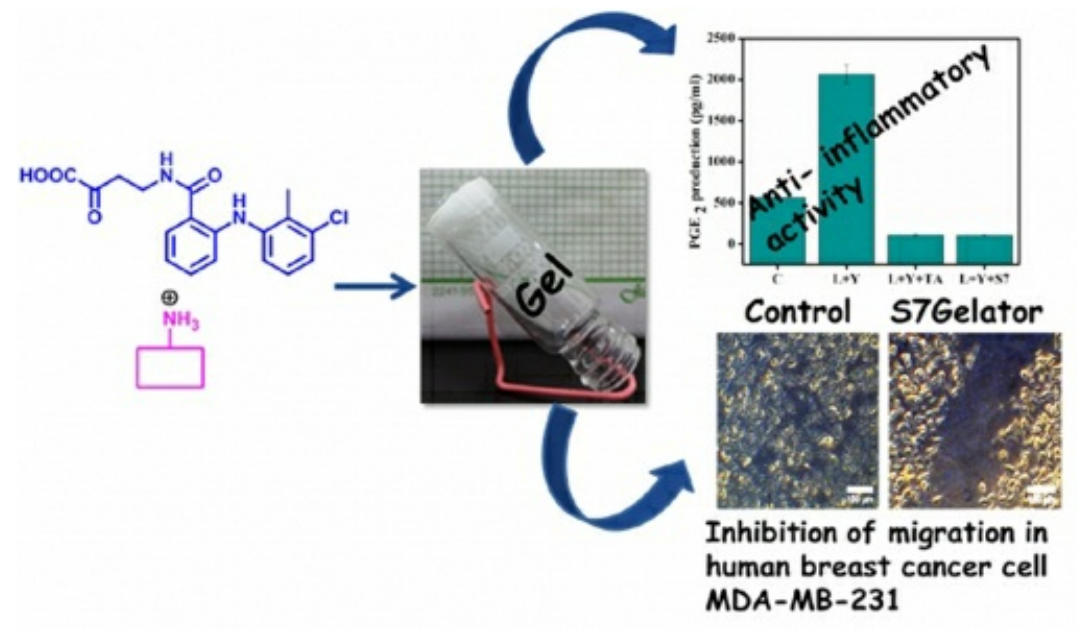

Keywords: Anti-inflammatory; Anti-cancer;SAFiNs 